\title{
Orientation unbound: Dissociation of identity and orientation under rapid serial visual presentation
}

\author{
Michael C. Corballis, Cole Armstrong, and Zhuoying Zhu \\ University of Auckland, Auckland, New Zealand
}

\begin{abstract}
Participants were shown rapid sequences of three letters, flanked by digits, each rotated $0^{\circ}, 90^{\circ}, 180^{\circ}$, or $270^{\circ}$ clockwise from upright. In Experiment 1, the participants tried to report the letter that matched the orientation of an arrow, presented either before (before task) or after (after task) the sequence. A third task (total task) required them to report all of the letters. Accuracy for individual letters was significantly better in the total task than in the before task, and better in the before task than in the after task, suggesting particular difficulty in binding orientation to identity. In Experiment 2, the participants were given letter probes and were asked to indicate the orientation of the probed letter. Although report was above chance, there were frequent illusory conjunctions. Since perception of orientation must depend on prior establishment of identity, our results suggest that orientation and identity may become unbound during processing and are held in parallel storage systems.
\end{abstract}

People can generally recognize familiar shapes regardless of their orientation in space (Corballis, 1988), suggesting that identity is dissociated from orientation in visual processing. This dissociation is exhibited in neurological patients with parietal damage who are able to recognize objects correctly but unable to determine their orientations (Fujinaga, Muramatsu, Ogano, \& Kato, 2005; Harris, Harris, \& Caine, 2002; Turnbull, Della Sala, \& Beschin, 2002). Conversely, patients with object agnosia have little difficulty in locating objects. The coding of shape independently of orientation seems to depend on the temporal lobe, and Quiroga, Reddy, Kreiman, Koch, and Fried (2005) have recently reported that single cells in the human medial temporal lobe respond to well-known individuals, landmarks, or objects shown from different viewpoints.

Indirect evidence for the dissociation of shape and orientation in normal participants has come from experiments on repetition blindness (RB). If familiar objects are presented in rapid serial visual presentation (RSVP) at rates of about $10 / \mathrm{sec}$, observers typically fail to notice repetitions of the same object but report having seen it only once. One interpretation is that each object may find a match in memory but that presentation is too fast for each presentation to be recorded as a separate, conscious event. That is, there is recognition of type, without token individuation (Kanwisher, 1987). Experiments have shown that RB occurs when the repeated objects differ in orientation (Harris \& Dux, 2005; Kanwisher, Yin, \& Wojciulik, 1999). The same is true of uppercase letters shown in different orientations; recognition of individual letters is independent of orientation, and RB occurs regardless of the angular distance between two presentations of the same letter (Corballis \& Armstrong, 2007).
Corballis and Armstrong (2007) also reported a striking deficit in the ability to report pairs drawn from the letters b, d, p, and q, presented in RSVP sequences, even though these letters are differently named. This result seemed to be a case of RB for the same shape presented in different orientations or different left-right versions. RB has also been shown for letters printed in different cases (Bavelier \& Potter, 1992; Corballis \& Armstrong, 2007), suggesting that it can depend on repetition of differently shaped stimuli with the same name. Taken together, these results indicate that RB can occur at different levels of processing - the level at which basic shape is extracted and the level at which the name of a shape is identified.

In the present experiments, we focused more specifically on the processing of orientation itself. As we have seen, both recognition and RB seem to be independent of orientation, at least in the case of letters or common objects. One possibility, suggested by Luck and Vogel (1997), is that items in visual short-term memory are stored as integrated wholes. This implies that although identity is established independently of orientation, the orientations should still be available in memory and bound to the identities. However, Wheeler and Treisman (2002) were unable to replicate Luck and Vogel's finding, and their work suggested instead that features from different dimensions are extracted independently and stored in parallel in short-term visual memory. For example, lines of varying orientation and color might be stored in parallel memory stacks, one holding the orientations and the other the colors. Focused attention is required to maintain the binding, and under stringent conditions, such as RSVP, one might expect little binding to occur. Hence, orientation may be extracted but remain unbound.

M. C. Corballis, m.corballis@auckland.ac.nz 
In the studies by Luck and Vogel (1997) and Wheeler and Treisman (2002), the stimuli were presented in spatial arrays, and their conclusions about storage may not apply to stimuli presented in rapid temporal sequence. In the case of rotated objects or letters, there is an added complication in that, logically, orientation cannot be determined until the stimulus is identified. There is some empirical support for this from a study showing that decisions about the orientations of letters take longer than decisions about their identity (Corballis, Zbrodoff, Shetzer, \& Butler, 1978), although this effect was possibly confounded by differences in the discriminability of shapes and orientations. Nevertheless, if the extraction of orientation does require an extra processing step, it is possible that orientation is not extracted at all under RSVP. That is, presentation may be too fast to allow processing to proceed beyond identification, so the orientation is not registered.

The experiments reported here were designed to assess the extent to which the orientations of rotated letters presented in RSVP are processed and available. The foregoing suggests three different possibilities. First, presentation may be too rapid to allow more than minimal processing of orientation, at best, so that only identity is extracted. Second, orientation may be extracted but held in parallel storage, as has been proposed by Wheeler and Treisman (2002). In this case, binding of orientation to identity should be minimal, at best. Third, both orientation and identity may be held in the same storage buffer, as has been proposed by Luck and Vogel (1997), and binding should be relatively straightforward. The technique we used was to probe for individual items in RSVP streams of three rotated letters, by using either single orientation probes and asking for report of the associated letter (Experiment 1) or single letter probes and asking for report of the associated orientation (Experiment 2). The probes were presented either before or after the sequences. In the case of postsequence probes, in particular, we would expect performance to be little better than chance if orientation is not extracted or is stored in a parallel store. On the other hand, if items are stored as integrated wholes, the matching of probes to items should be relatively straightforward.

Although the use of letters as stimuli may limit the generality of our findings, we chose letters for several reasons. First, they are highly overlearned and, therefore, rapidly and easily recognized and named. Second, they are twodimensional, so that changes in orientation are not complicated by the third dimension, whereas three-dimensional rotation of solid objects results in variations in featural access - or access to geons, in the terminology of Biederman and Gerhardstein (1993). Third, there is no ambiguity as to the standard, or "canonical," view, whereas in the case of three-dimensional objects there is some question as to whether the canonical view should be a frontal view, a side view, or a three-quarters view. It might be argued that we already know that letters are quickly recognized regardless of orientation, although departures from orientation can result in a slight loss of speed or accuracy (Corballis et al., 1978; Jolicœur \& Landau, 1984). However, the focus here is not on whether letters can be identified regardless of orientation, but on the processing of orientation itself.
A further possible limitation of our study is that the orientations were restricted to the canonical directionsnamely, $0^{\circ}, 90^{\circ}, 180^{\circ}$, or $270^{\circ}$ clockwise from the upright. There were two main reasons for this. First, these orientations are distinct and easily categorized and can, therefore, serve as unambiguous probes. Second, use of these orientations largely removes any complications associated with the so-called oblique effect (Appelle, 1972). The letters we used as stimuli (see the Method section) include the same proportions of oblique and nonoblique lines under each of the canonical rotations.

\section{EXPERIMENT 1}

\section{Method}

Participants. Eight women and 7 men between the ages of 19 and 34 years volunteered as participants. The mean age was 26 years.

Stimuli. The target stimuli were chosen from the uppercase letters A, F, J, L, P, R, V, T, and Y. The participants were shown the set of letters in advance of the experimental trials. The digits 2 through 9 were used as masks. Each stimulus subtended a visual angle of $1.5^{\circ}$ in height and approximately $1^{\circ}$ in width.

Each RSVP sequence comprised one digit, followed by three letters, and then a final digit, all chosen randomly without replacement. Each character was on the screen for seven screen refreshes, or approximately $117 \mathrm{msec}$, and was presented in the center of the screen at $0^{\circ}, 90^{\circ}, 180^{\circ}$, or $270^{\circ}$ clockwise from the upright. The orientations across the three letters were systematically permuted so that the same orientation of a letter was never repeated within a sequence. This amounted to 24 different possible permutations. Since each letter position was probed equally often at each orientation, there were a total of 72 sequences per run. The stimuli were white against a dark gray background, and a small white cross in the center of the screen served as a fixation point prior to presentation of the stimuli.

Procedure. There were three tasks, which the participants performed in counterbalanced order. In each task, the 72 five-character streams were presented in random order, and the participants initiated presentation of each stream by pressing the space bar. The tasks were as follows.

1. Before task. Each trial began with the fixation cross, and a 500-cps warning tone for $200 \mathrm{msec}$. The cross then disappeared, and a probe arrow $1.5^{\circ}$ long appeared on the screen for $100 \mathrm{msec}$. The stream was presented $500 \mathrm{msec}$ later. The arrow pointed $0^{\circ}, 90^{\circ}$, $180^{\circ}$, or $270^{\circ}$ clockwise from vertical to indicate which letter the participants were to report. Each position and each orientation were probed equally often. After the stream had appeared, a prompt on the screen instructed the participants to type in the letter that matched the orientation of the probe.

2. After task. In this case, the probe arrow appeared for $500 \mathrm{msec}$ $200 \mathrm{msec}$ after presentation of the final digit in the stream. There was again a 500 -cps warning tone, followed $500 \mathrm{msec}$ later by the stream. A prompt after the probe arrow instructed the participants to type in the letter that matched the orientation of the probe.

3. Total task. In this case, there were no probes. The stream appeared $500 \mathrm{msec}$ after the warning tone, and immediately following the stream, the participants were prompted to type in all three letters in the stream. The digits were to be ignored.

The tasks were counterbalanced across participants, with 5 participants in each of the cyclic combinations of before, after, and total tasks. In all three tasks, the participants were explicitly instructed not to report digits.

\section{Results}

In all the subsequent analyses, where violations of the assumption of sphericity occur, significance levels are corrected according to the Greenhouse-Geisser correction. 


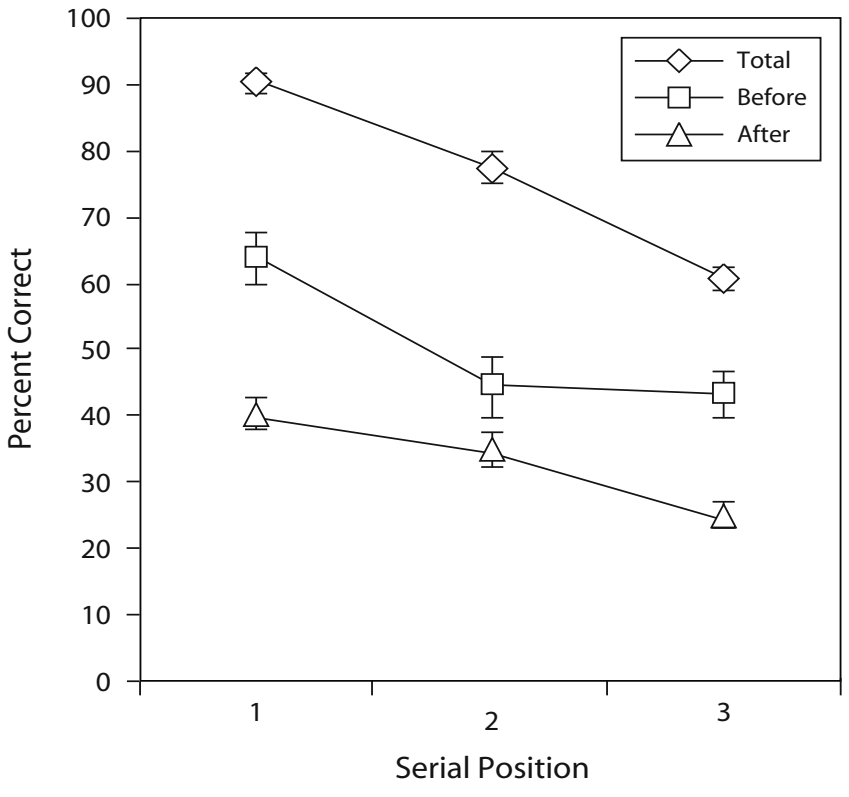

Figure 1. Percent correct for each serial position in each task in Experiment 1. Error bars are standard errors.

Analysis of accuracy. In a first analysis, a response was scored as correct in the probe tasks only if it matched the letter in the stream whose orientation matched that of the probe. In the total task, letters were scored correct if they matched those presented regardless of the order of report.

A repeated measures ANOVA with task, letter position, and letter orientation as within-subjects factors and task order as a between-subjects factor revealed main effects of task $[F(2,24)=193.49, p<.001]$ and letter position $[F(2,24)=36.61, p<.001]$, as well as a significant interaction between task and letter position $[F(4,48)=3.72, p=$ $.035]$. Figure 1 shows the mean percent correct for each serial position for each task; the main contributor to the interaction was a more pronounced primacy effect for the before task than for the other two tasks, which is not surprising since the participants could stop processing the sequence if the probe matched the first letter in the sequence.

The main effect of orientation was not significant $[F(3,36)=1.47, p=.245]$, and orientation did not interact significantly with any other factor ( $p \geq .324)$. Figure 2 plots percent correct for each orientation for each task. For the total task, the orientation function is virtually flat, suggesting that identity is extracted independently of orientation. For the probe tasks, accurate performance depends on the orientation of the probe itself, as well as on the orientation of the probed letter, but again there is little effect of orientation. That is, the orientation of the probe itself had little effect on performance. One might have expected the participants to have been better able to pick out the upright letter than those in other orientations, but there was little evidence that this was so-except perhaps in the after task, but even here the effect of orientation was not significant $[F(3,36)=1.82, p=.164]$.
Analysis of relation between probed and reported positions. Accuracy in the after task was $33.4 \%$, which is almost exactly what one would expect if the participants had simply chosen randomly among the three presented letters. In order to determine whether there was a systematic relation between the probed letter and the reported letter, we therefore undertook a second analysis in which reported letters in the probe tasks were scored as correct so long as they were in the presented set, regardless of whether their orientations matched the probe or not. Because the relation of the reported letter to the probed letter was the primary focus of this analysis, the total task was not included.

A repeated measures ANOVA with task, probed position, reported letter position, and letter orientation as withinsubjects factors and order as a between-subjects factor resulted in significant main effects of task $[F(1,12)=9.29$, $p=.01]$ and probed letter position $[F(2,24)=10.95, p<$ $.001]$. There was again no significant effect of orientation $[F(3,36)=0.24, p=.791]$, and orientation did not interact significantly with any other factor $(p>.277)$.

There was a significant interaction between probed position and reported letter position $[F(4,48)=23.49, p<$ $.001]$ and between task, probed position, and reported letter position $[F(4,48)=17.23, p<.001]$. As is shown in Table 1, the participants generally reported the probed letter more often than the nonprobed letters; the interaction between probed position and reported letter position was highly significant for the before task $[F(4,48)=32.67, p<$ $.001]$ but just failed to reach significance for the after task $[F(4,48)=2.82, p=.051]$. The simple effect of position was computed for each probe on both tasks and then broken down into paired comparisons of the probed letter versus the other two. Scheffé comparisons showed that the probed letter was reported significantly $(p<.05)$ more accurately than the other two letters only for the first two positions in

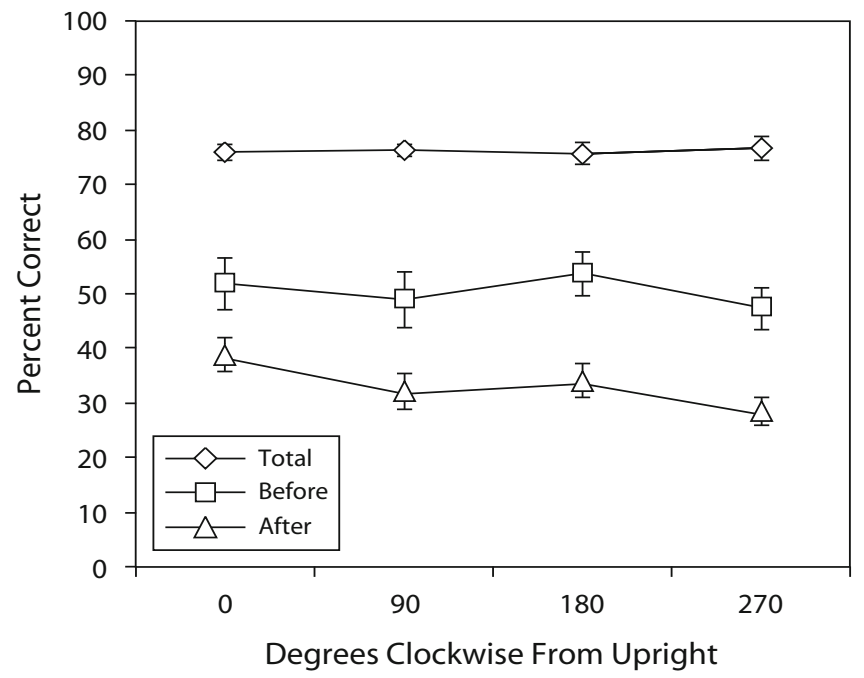

Figure 2. Percent correct for each orientation for each task in Experiment 1. Error bars are standard errors. 
Table 1

Mean Percentages of Letters Reported As a Function of Task, Position of Probed Letter, and Position of Reported Letter in Experiment 1

\begin{tabular}{|c|c|c|c|c|c|c|c|c|}
\hline \multirow{3}{*}{$\begin{array}{c}\text { Reported } \\
\text { Letter Position }\end{array}$} & \multicolumn{4}{|c|}{ Before Task } & \multicolumn{4}{|c|}{ After Task } \\
\hline & \multicolumn{3}{|c|}{ Probed Letter Position } & \multirow[b]{2}{*}{ Mean } & \multicolumn{3}{|c|}{ Probed Letter Position } & \multirow[b]{2}{*}{ Mean } \\
\hline & 1 & 2 & 3 & & 1 & 2 & 3 & \\
\hline 1 & $63.6^{*}$ & 27.8 & 21.9 & 37.8 & $40.3^{*}$ & 28.9 & 30.3 & 33.2 \\
\hline 2 & 16.9 & $44.4^{*}$ & 27.5 & 29.6 & 29.4 & 35.0 & 30.6 & 31.7 \\
\hline 3 & 11.7 & 21.7 & 43.3 & 25.6 & 20.3 & 21.4 & 25.0 & 22.2 \\
\hline Total & 92.2 & 93.9 & 92.7 & 92.9 & 90.0 & 85.3 & 85.9 & 87.1 \\
\hline
\end{tabular}

Note-Asterisks show means that differ significantly $(p<.05)$ from both of the other means in the same column. No other paired comparisons reach the .05 level of significance.

the before task and only for the first position in the after task. These effects are indicated by asterisks in Table 1.

Table 1 also shows that, when probe position was disregarded, the participants reported one of the presented letters on $92.9 \%$ of the trials on the before task and $87.1 \%$ of the trials on the after test. These high values mean that the participants seldom failed to report a letter from the presented set but often reported the wrong one.

\section{Discussion}

First, as in the earlier studies of report of letters under RSVP (Corballis \& Armstrong, 2007), there was no evidence that the orientations of the letters had any effect on report. This was so regardless of whether individual letters were probed or whether total report was attempted. The earlier finding of RB regardless of the orientations of the repeated letters raised the possibility that, under RSVP, observers may be unable to process the orientations of letters at all. The present experiment indicates, however, that at least some processing of orientation must have been possible, at least in the before task, since the participants reported the probed letter more often than they reported unprobed letters. Nevertheless, their ability to report the probed letter was actually worse than their ability to report all three letters. This was so even when the orientation was given prior to presentation of the sequence (before task) and even when the probed item was the first one in the sequence (see Figure 1) and the participants need not have processed the letters that occurred later in the sequence. These findings suggest that it is more difficult to process the identity and orientation of a single one of three letters presented under RSVP than it is to process just the identities of all three letters. This argues against the possibility that the stimuli were held in short-term visual memory as integrated wholes, with orientation bound to identity, as has been suggested by Luck and Vogel (1997).

Logically, the orientation of a letter cannot be established until its identity is known. This implies that processing of both identity and orientation should take longer than the processing of identity alone. This could explain why report of the probed letter rose above that of unprobed letters only for the first two letters in the case of the before task and only for the first letter in the case of the after task. That is, the participants may have adopted a strategy of extracting orientation as well as identity, thereby slowing overall processing. Nevertheless, when report on the probe tasks was scored regardless of the letter probed, accuracy on the probe tasks rose above that on total report, indicating that the extra requirement to process orientation did not greatly jeopardize the processing of identity.

Although performance on the probe tasks was above chance, at least for early items in the sequence, there were frequent reports in which the reported letter was in the sequence but did not match the probe. This suggests either that orientation was not processed or that orientations and identities were held in separate short-term memory stores (Wheeler \& Treisman, 2002), so that the choice of letter was based on random selection from the store of identities, giving rise to illusory conjunctions (Treisman, 1988). In Experiment 2, we reversed the design of Experiment 1. Instead of providing orientation probes and testing for the associated letters, we presented letter probes to the participants and tested for the associated orientations. On trials on which participants failed to associate a letter with its orientation, the question is whether they chose at random from the four possible orientations or whether they chose from the three orientations that were actually present in the sequence, implying illusory conjunctions.

\section{EXPERIMENT 2}

\section{Method}

Participants. Eight women and 7 men between the ages of 19 and 34 years volunteered as participants.

Stimuli. The sequences were essentially the same as those in Experiment 1 , except that they consisted of two digits, followed by three letters and then two digits, and each character was on the screen for six screen refreshes, or approximately $100 \mathrm{msec}$. The extra digits and the more rapid presentation were introduced to make the task slightly more difficult, since there were only four choice alternatives in this experiment. The sequences of digits and letters were the same as those in Experiment 1, except that the probes were letters drawn from the presented sequence.

Procedure. There were again three tasks, each consisting of 72 sequences, presented in counterbalanced order. In the before task, each trial began with the fixation cross and a 500-cps warning tone for $200 \mathrm{msec}$. The cross then disappeared, and a probe letter appeared on the screen for $100 \mathrm{msec}$. The stream was presented $500 \mathrm{msec}$ later. Each position and each orientation were probed equally often. After the stream had appeared, a prompt on the screen instructed the participants to indicate the orientation of the probed letter, using the $3 \times 3$ numeral keys on the right of the keyboard. The 2, 4, 6, and 8 keys showed arrows pointing down, leftward, rightward, and up, respectively. In the after task, the probe letter appeared for $500 \mathrm{msec}$, $200 \mathrm{msec}$ after presentation of the final digit in the stream. There 


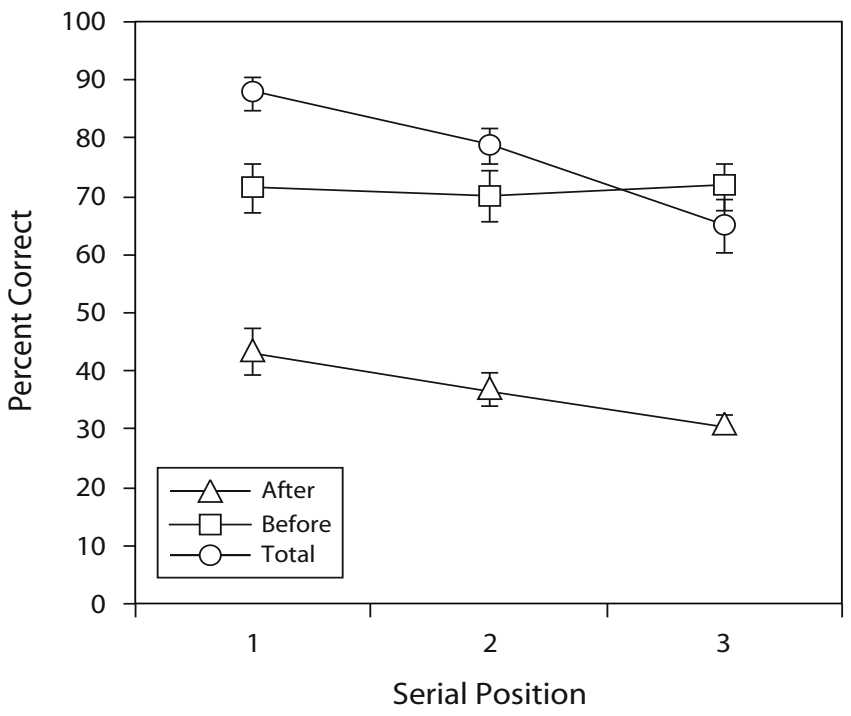

Figure 3. Percent correct in report for each serial position in each task in Experiment 2. For the total task, report is of letters, whereas for the before and after tasks report is of probed orientations. Error bars are standard errors.

was again a 500 -cps warning tone, followed $500 \mathrm{msec}$ later by the stream. A prompt after the probe letter instructed the participants to press the arrow key that matched the orientation of the probe. If unable to choose, the participants pressed the space bar. A total task was also included in which the participants were asked simply to report the three letters when the prompt appeared.

\section{Results}

In all the subsequent analyses, where violations of the assumption of sphericity occur, significance levels are corrected according to the Greenhouse-Geisser correction.

Total task. First, report of letters in the total task was analyzed. Reported letters were scored as correct if they corresponded to those in the presented stream, regardless of order of report. An ANOVA with position and orientation as within-subjects factors and order as a betweensubjects factor showed only a significant effect of position $[F(2,24)=21.93, p<.001$; see Figure 3]. As in Experiment 1 , there was a strong primacy effect. The effect of orientation was not significant $[F(3,36)=2.14, p=.112]$ and is plotted in Figure 4. If anything, report is lowest for the upright orientation.

Probe tasks: Accuracy. For the two probe tasks, the first analysis considered correct responses only. An ANOVA was carried out with order as a between-subjects factor and task, position, and orientation as within-subjects factors. This revealed a highly significant advantage of the before task over the after task $[F(1,12)=84.56, p<.001]$. There were also significant interactions between task and position $[F(2,24)=3.60, p=.043]$ and task and orientation $[F(3,36)=4.75, p=.002]$. Percentage correct for each task is shown as a function of position in Figure 3 and of orientation in Figure 4. The effect of orientation failed to reach significance for either the before task $[F(3,36)=$ 2.536, $p=.072]$ or the after task $[F(3,36)=2.15, p=$ $.111]$, so the interaction will not be considered further.
The serial position effect was not significant for the before task $[F(2,24)=.216, p=.815]$ but was significant for the after task $[F(2,24)=3.92, p=.034]$, with trend analysis revealing a (downward) linear trend $[F(1,24)=$ 7.72, $p=.017]$.

We have plotted the results for all three tasks in Figures 3 and 4, despite the fact that report of letters was required in the total task and report of orientations in the other two tasks, so that accuracy is not strictly comparable in the two cases. Chance should have strongly favored the two probe tasks, since there were only four orientations to choose from and the task required report of only one orientation, whereas the total task required report of all three letters. Even so, the participants were at least as accurate at reporting all three letters as at reporting the orientation of just one of the letters in the before task, and were considerably more accurate than in the after task.

Probe tasks: Orientation confusions. For the next analysis, we classified all responses according to correct orientation and the reported orientation, as well as according to the position of the probed letter. An ANOVA included probe position, orientation of the probed letter, orientation of the reported letter, and task.

There were no significant effects associated with position ( $p \geq .211)$. The only significant effects were the interactions between task and reported orientation $[F(3,36)=$ $3.72, p=.036]$, correct orientation and reported orientation $[F(9,108)=79.32, p<.001]$, and task, correct orientation, and reported orientation $[F(9,108)=31.75, p<$ $.001]$. Since the triple interaction was significant, separate analyses were carried out for each task.

For the before task, there was a significant effect of reported orientation $[F(3,36)=5.73, p=.006]$; Scheffé tests showed that reports of $0^{\circ}$ were significantly $(p<.05)$ fewer than those of $90^{\circ}$ or $270^{\circ}$, reflecting a bias against report of $0^{\circ}$ and perhaps a bias toward reporting $90^{\circ}$ and

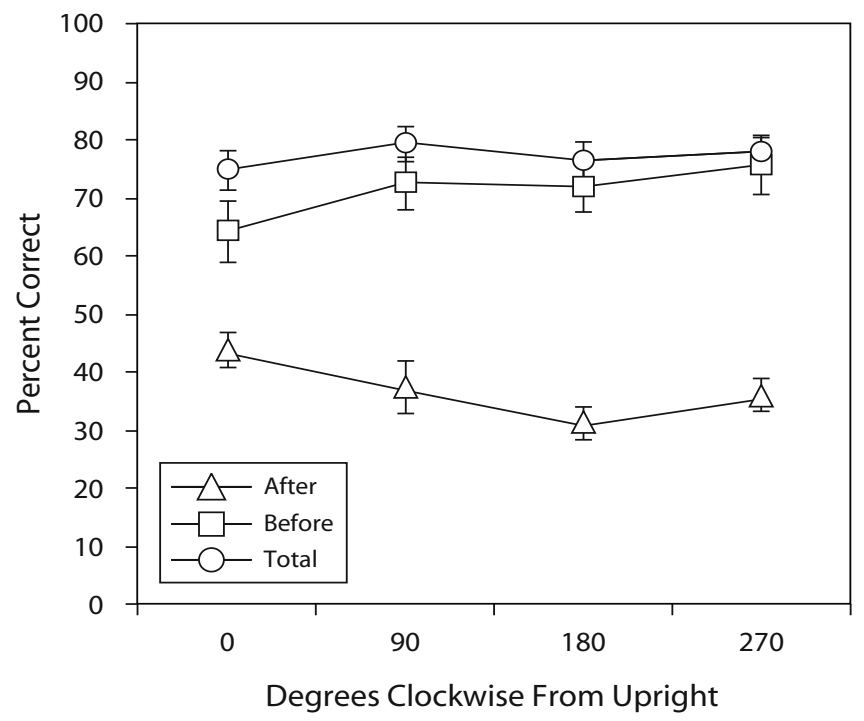

Figure 4. Percent correct for each task in Experiment 2. For the total task, report is of letters, whereas for the before and after tasks report is of probed orientations. Error bars are standard errors. 
Table 2

Mean Percentages of Orientations Reported As a Function of Task, Correct Orientation, and Reported Orientation in Experiment 2

\begin{tabular}{|c|c|c|c|c|c|c|c|c|c|c|}
\hline \multirow{3}{*}{$\begin{array}{c}\text { Reported } \\
\text { Orientation }\end{array}$} & \multicolumn{5}{|c|}{ Before Task } & \multicolumn{5}{|c|}{ After Task } \\
\hline & \multicolumn{4}{|c|}{ Orientation of Probed Letter } & \multirow[b]{2}{*}{ Mean } & \multicolumn{4}{|c|}{ Orientation of Probed Letter } & \multirow[b]{2}{*}{ Mean } \\
\hline & $0^{\circ}$ & $90^{\circ}$ & $180^{\circ}$ & $270^{\circ}$ & & $0^{\circ}$ & $90^{\circ}$ & $180^{\circ}$ & $270^{\circ}$ & \\
\hline $0^{\circ}$ & $64.1^{*}$ & 7.0 & 6.7 & 4.1 & $20.45^{\dagger}$ & $43.7^{*}$ & 23.3 & 25.2 & 21.5 & 28.43 \\
\hline $90^{\circ}$ & 11.1 & $72.6^{*}$ & 10.7 & 13.0 & 26.85 & 22.2 & $37.4+$ & 23.0 & 23.3 & 26.48 \\
\hline $180^{\circ}$ & 11.9 & 5.6 & $71.9^{*}$ & 7.4 & 24.20 & 15.6 & $18.2 \ddagger$ & 31.1 & $19.3 \ddagger$ & 21.05 \\
\hline $270^{\circ}$ & 13.0 & 14.8 & 10.7 & $75.6^{*}$ & 28.53 & 18.5 & 21.1 & 20.7 & $35.9 末$ & 24.05 \\
\hline
\end{tabular}

${ }^{*}$ Differs significantly $(p<.05)$ from other values in the same column. ${ }^{\dagger}$ Differs significantly $(p<.05)$

from reports of $90^{\circ}$ and $270^{\circ}$. $¥$ Values that differ significantly $(p<.05)$ in the same column.

$270^{\circ}$. These biases are also evident in the accuracy scores shown in the diagonal entries of Table 2 . There was also a significant interaction between correct orientation and reported orientation $[F(9,108)=88.8, p<.001]$. For each probed orientation, Scheffé tests showed only that participants were significantly $(p<.001)$ more likely to report the correct orientation than to report unprobed ones.

For the after task, the effect of reported orientation was not significant $[F(3,36)=1.19, p=.323]$, although in this case the participants were most likely to respond $0^{\circ}$, in contrast to the bias against $0^{\circ}$ in the before task. The interaction between correct and reported orientation was significant $[F(9,108)=9.57, p<.001]$, again reflecting more accurate report of the probed orientation than of unprobed ones (see Table 2). However, Scheffé tests for each probed orientation revealed mixed results. For $0^{\circ}$, the participants responded correctly significantly $(p<.05)$ more often than they reported the other three orientations, which did not differ significantly. For $90^{\circ}$, correct reports were significantly more frequent only than the number of reports of $180^{\circ}$. For $180^{\circ}$, there were no significant differences among the reported orientations. For $270^{\circ}$, correct reports were significantly more frequent $(p<.05)$ than reports of $180^{\circ}$.

Probe tasks: Position confusions. In order to test for possible illusory conjunctions, we next scored the responses according to the position of the letter that matched the reported orientation. Since there were only three presented orientations, the participants sometimes responded with an orientation that was not among those presented. The mean percentages of orientations reported as a function of task, probed orientation position, and reported orientation position are shown in Table 3. An ANOVA of the reports as a function of task, probed position, and reported position necessarily revealed no effect of task or of probed position, since all reports were accounted for, but there was a main effect of reported probe position $[F(3,36)=87.19$, $p<.001]$. Scheffé tests showed that the percentage of trials on which the response orientation did not match any of the actual orientations differed significantly $(p<.001)$ from each of those that did match, and the main effect was no longer significant when these reports were removed $[F(2,24)=2.83, p=.084]$.

More important, there were significant interactions between probed position and reported position $[F(6,72)=$ $81.15, p<.001]$ and between task, probed position, and reported position $[F(6,72)=45.40, p<.001]$. These interactions remained significant when the report of orientations not in the stream were removed $[F(4,48)=91.42$ and 51.39, respectively; $p<.001]$ and reflect the fact that the reported orientation position generally matched the probed orientation position but that this varied between tasks (the data are shown for each task in Table 3). The interaction between reported and probed orientation positions was highly significant for the before task $[F(4,48)=$ 101.96, $p<.001]$ and also significant for the after task $[F(4,48)=13.82, p<.001]$.

It is clear from Table 3 that, in the before task, the reported position matched the probed position on $70 \%$ or more of the trials, suggesting reasonably accurate binding of orientation to letter, although there were also many false conjunctions. Accuracy was considerably lower for the after task. For the before task, Scheffé tests at each probed position, again omitting the report of orientations from outside the stream, showed that matching orientations were more often reported than nonmatching ones ( $p \leq .020)$, which did not differ significantly from each other, with one exception: When the

Table 3

Mean Percentages of Orientations Reported As a Function of Task, Probed Orientation Position, and Position of the Letter With the Reported Orientation

\begin{tabular}{|c|c|c|c|c|c|c|c|c|}
\hline \multirow{3}{*}{$\begin{array}{c}\text { Position of } \\
\text { Reported Orientation }\end{array}$} & \multicolumn{4}{|c|}{ Before Task } & \multicolumn{4}{|c|}{ After Task } \\
\hline & \multicolumn{3}{|c|}{ Probed Position } & \multirow[b]{2}{*}{ Mean } & \multicolumn{3}{|c|}{ Probed Position } & \multirow[b]{2}{*}{ Mean } \\
\hline & 1 & 2 & 3 & & 1 & 2 & 3 & \\
\hline 1 & $71.4^{*}$ & 14.4 & 12.8 & 32.87 & $43.3^{*}$ & 23.6 & 24.4 & 30.43 \\
\hline 2 & $16.1^{\dagger}$ & $70.0^{*}$ & 10.3 & 32.13 & 20.3 & $36.9^{*}$ & 26.7 & 27.97 \\
\hline 3 & 7.5 & 11.7 & $71.7^{*}$ & 30.30 & 22.8 & 23.6 & 30.8 & 25.67 \\
\hline Not present & 5.0 & 3.9 & $5.3 \ddagger$ & 4.73 & $13.7 \ddagger$ & 15.8 & $8.1+$ & 15.87 \\
\hline
\end{tabular}

*Differs significantly $(p<.014)$ from report of nonmatching orientations present in the stream. $\dagger$ Differs significantly from the other nonmatching orientation present in the stream. significantly from the nonmatching orientations present in the stream. 
probed orientation was in Position 1, the participants significantly more often reported the orientation in Position 2 than the one in Position $3(p=.024)$. For the after test, Scheffé tests at each probed position showed that the orientation of the probed position was significantly $(p<.05)$ more often reported than the orientations of the other positions for the first and second positions, but not for the third.

If the participants had responded at random, the expected proportion of reports would have been $25 \%$ for each orientation, including the one not represented in the stream. When the participants erred, they were significantly more likely to report one of the two incorrect orientations that appeared in the stream than to report the one not in the stream $(p \leq .014)$, and with the exception reported in the previous paragraph, the report of the two incorrect letters in the stream did not differ significantly. That is, errors could generally be classed as illusory conjunctions, rather than as random responses.

\section{Discussion}

Although accuracy on this task cannot be compared directly with that in Experiment 1, comparing significance levels between experiments suggests better performance in Experiment 2. In Experiment 2, letter probes resulted in a significantly better report of orientation for all three positions in the before task and for the first two positions in the after task (Table 2). In Experiment 1, orientation probes resulted in a significantly better report of the letter in only the first two positions in the before task and in only the first position in the after task (Table 1). Moreover, the interaction between actual and reported positions, reflecting overall accuracy, was significant only for the before task in Experiment 1 but was highly significant for both tasks in Experiment 2.

A possible explanation for these differences derives from the fact that the identity of a letter must be established before its orientation can be determined. When presented with an orientation probe, therefore, search for the matching letter is slowed by the need to establish both identity and orientation. When presented with a letter probe, however, the participant need only process identity until the matching letter is found and then process the orientation for that letter alone. Even so, the results of Experiment 2 suggest poor binding of orientation to identity, especially when the probe was presented after the sequence.

A critical finding was that the participants were significantly more likely to respond with one of the presented orientations than with the (one) orientation that did not appear in the stream, even when the probe letter appeared after the stream (see Table 3). This suggests that the participants did not merely respond by choosing among the four orientations at random when they were unsure of a match. Rather, the orientations of the letters must have been processed, even if often not correctly matched with the letters themselves. This suggests, in turn, that the report of nonmatching letters in Experiment 1 was not due simply to the absence of orientation information and random selection among the letters but, rather, that the participants stored the identities and orientations in separate files, as has been proposed by Wheeler and Treisman (2002).

\section{GENERAL DISCUSSION}

The results confirm the conclusion from an earlier study on RB with letters (Corballis \& Armstrong, 2007), as well as those with pictures of objects (Harris \& Dux, 2005; Kanwisher et al., 1999), that participants process the identity independently of angular orientation under conditions of RSVP. This is not to say, however, that identity is extracted independently of orientation for all kinds of patterns. For example, Tarr and Pinker (1989) found evidence of orientation dependence and a role for mental rotation in the processing of novel shapes, although Wilson and Farah (2003) have shown that orientation dependence for such shapes depends partly on top-down control processes and partly on stimulus features. There has been debate as to whether rotational invariance for familiar objects is achieved through decomposition into orientation-independent geons (Biederman \& Gerhardstein, 1993) or through familiarity with multiple viewpoints (Tarr \& Bülthoff, 1995). The dissociation between identity and orientation shown in the present experiments argues against the notion that familiar patterns are stored in multiple views, at least in the case of letters.

In the introduction, we distinguished three possibilities concerning the processing of orientation: (1) RSVP would severely limit the processing of orientation, perhaps to the point that the orientation would not be processed at all; (2) orientation would be processed but stored separately from identity (as has been proposed by Wheeler \& Treisman, 2002) and so remain largely unbound from identity; and (3) stimuli would be stored as integrated wholes (as proposed by Luck \& Vogel, 1997), so that identity and orientation would remain bound. The results seem most consistent with the second of these possibilities; that is, identity and orientation were to a large extent unbound and held in parallel short-term stores, as has been proposed by Wheeler and Treisman. This is most apparent in Experiment 2, where the participants were asked to provide the orientations associated with the letter probe. As is shown in Table 3, they were significantly more likely to report the orientation of one of the letters in the sequence, even if it did not match the probe, than to report the orientation that was not in the sequence. When the pressure on binding was most extreme, as when a postsequence probe was the third letter in the sequence, the choice of the associated orientation from among the three presented orientations failed to exceed chance but was still significantly higher than the choice of the orientation that was not in the sequence.

There was also evidence for the first of the possibilities above. That is, although there was some parallel storage of orientation and identity, the results suggest that extraction of orientation was difficult. In Experiment 1, for example, the participants were less accurate at reporting letters probed by orientation than at reporting all three letters. Even in Experiment 2, total report of letters was better than report of single probed orientations. Although report of orientations is not strictly comparable to report of letters, chance should favor report of orientations, since there were only four orientations to choose from. The likely reason that extraction of orientation was difficult is that the orientation of a letter cannot be established until the identity of the letter is 
known. Under RSVP, there is little time to proceed to the extra step of extracting orientation. In this respect, the present study differs from those of Luck and Vogel (1997) and Wheeler and Treisman (2002). In their studies, there were no time constraints limiting the extraction of orientation, and the stimuli were simply lines whose orientations could be extracted in parallel with other features.

In an fMRI study, Valyear, Culham, Sharif, Westwood, and Goodale (2006) have shown that the dorsal stream in the occipito-parietal cortex is responsive to changes in the orientation of objects but impervious to changes in identity, whereas the ventral stream in the temporo-occipital cortex is responsive to changes in identity but not in orientation. Nevertheless, if the identity of an object is confined to the ventral stream, the dorsal stream cannot "know" its orientation, since orientation depends on identity. Rather, it may simply encode the manner in which the shape is located in space and how it fills that local region of space (cf. Ungerleider \& Mishkin, 1982). This information may allow an observer to navigate with respect to the shape, to grasp or trace it, to insert it into an appropriately shaped slot, but not to actually name it or identify it as familiar (Milner \& Goodale, 1995). Perception of the orientation of the shape itself must somehow depend on the mapping of the shape, as identified by the ventral system, onto the spatial template supplied by the dorsal stream. Precisely how this is done remains unclear, but the process is presumably attention demanding (cf. Treisman, 1988; Wheeler \& Treisman, 2002), time consuming, and greatly attenuated under RSVP.

In summary, our results suggest that letters are identified under RSVP largely independently of their orientations. This is consistent with so-called sparse coding (Vinje \& Gallant, 2000) in the ventral system, whereby identity is extracted independently of such circumstantial attributes as orientation, location, viewpoint, and so forth (cf. Quiroga et al., 2005). Nevertheless, our data suggest that orientation information is not entirely lost under RSVP. Rather, the data seem better characterized by a failure of binding, whereby there are frequent cases of illusory conjunctions. Given an orientation probe, participants often reported a letter in the sequence that did not match the probe, and given a letter probe, they often reported an orientation that was in the sequence but did not match the probe. Since the processing of orientation logically requires identity to have been established, it appears that orientation becomes unbound in the course of processing and/or retrieval.

\section{AUTHOR NOTE}

We thank Irina Harris and Jeff Hamm for discussions. Correspondence should be addressed to M. C. Corballis, Department of Psychology, University of Auckland, Private Bag 92019, Auckland 1001, New Zealand (e-mail: m.corballis@auckland.ac.nz).

\section{REFERENCES}

Appelle, S. (1972). Perception and discrimination as a function of stimulus orientation: The "oblique effect" in man and animals. Psychological Bulletin, 78, 266-278.
Bavelier, D., \& Potter, M. C. (1992). Visual and phonological codes in repetition blindness. Journal of Experimental Psychology: Human Perception \& Performance, 18, 134-147.

Biederman, I., \& Gerhardstein, P. C. (1993). Recognizing depthrotated objects: Evidence and conditions for three-dimensional viewpoint invariance. Journal of Experimental Psychology: Human Perception \& Performance, 19, 1162-1182.

Corballis, M. C. (1988). Recognition of disoriented shapes. Psychological Review, 95, 115-123.

Corballis, M. C., \& Armstrong, C. (2007). Repetition blindness is orientation blind. Memory \& Cognition, 35, 372-380.

Corballis, M. C., Zbrodoff, N. J., Shetzer, L. I., \& Butler, P. B. (1978). Decisions about identity and orientation of rotated letters and digits. Memory \& Cognition, 6, 98-107.

Fujinaga, N., Muramatsu, T., Ogano, M., \& Kato, M. (2005). A three-year follow-up of "orientation agnosia." Neuropsychologia, 43, $1222-1226$

HARRIS, I. M., \& Dux, P. E. (2005). Orientation-invariant object recognition: Evidence from repetition blindness. Cognition, 95, 73-93.

Harris, I. M., Harris, J. A., \& CAINE, D. (2002). Object orientation agnosia: A failure to find the axis? Journal of Cognitive Neuroscience, 13, 800-812.

Jolicceur, P., \& Landau, M. J. (1984). Effects of orientation on the identification of simple visual patterns. Canadian Journal of Psychology, 38, 80-93.

KANWISHER, N. (1987). Repetition blindness: Type recognition without token individuation. Cognition, 37, 117-143.

Kanwisher, N., Yin, C., \& WoJCIUlik, E. (1999). Repetition blindness for pictures: Evidence for the rapid computation of abstract visual descriptions. In V. Coltheart (Ed.), Fleeting memories: Cognition of brief visual stimuli (pp. 119-150). Cambridge, MA: MIT Press.

LuCK, S. J., \& Vogel, E. K. (1997). The capacity of visual working memory for features and conjunctions. Nature, 390, 279-281.

Milner, A. D., \& Goodale, M. A. (1995). The visual brain in action. New York: Oxford University Press.

Quiroga, R. Q., Reddy, L., Kreiman, G., \& Fried, I. (2005). Invariant visual representation by single neurons in the human brain. Nature, 435, 1102-1107.

TARR, M. J., \& BÜlthoff, H. H. (1995). Is human object recognition better described by geon structural descriptions or by multiple views? Comment on Biederman and Gerhardstein (1993). Journal of Experimental Psychology: Human Perception \& Performance, 21, 14941505.

TARR, M. J., \& PinKer, S. (1989). Mental rotation and orientation dependence in shape recognition. Cognitive Psychology, 21, 233-282.

Treisman, A. M. (1988). Features and objects: The fourteenth Bartlett memorial lecture. Quarterly Journal of Experimental Psychology, 40A, 201-237.

Turnbull, O. H., Della Sala, S., \& Beschin, N. (2002). Agnosia for object orientation: Naming and mental rotation evidence. Neurocase, 8, 296-305.

UnGerleider, L. G., \& MishKin, M. (1982). Two cortical visual systems. In D. J. Ingle, R. J. W. Mansfield, \& M. S. Goodale (Eds.), The analysis of visual behavior (pp. 549-586). Cambridge, MA: MIT Press.

Valyear, K. F., Culham, J. C., Sharif, N., Westwood, D., \& Goodale, M. A. (2006). A double dissociation between sensitivity to changes in object identity and object orientation in the ventral and dorsal visual streams: A human fMRI study. Neuropsychologia, 44, 218-228.

Vinje, W. E., \& Gallant, J. (2000). Sparse coding and decorrelation in primary visual cortex during natural vision. Science, 287, 12731276.

Wheeler, M. E., \& Treisman, A. M. (2002). Binding in short-term visual memory. Journal of Experimental Psychology: General, 131, 48-64.

Wilson, K. D., \& FARAH, M. J. (2003). When does the visual system use viewpoint-invariant representations during recognition? Cognitive Brain Research, 16, 399-415.

(Manuscript received April 12, 2006; revision accepted for publication July 27, 2006.) 